\title{
Development of low cost optical fiber kit to promote fiber optics at the school level
}

Phalguni Mathur, Parveen Jha, Deepak Kumar, Ajay Acharya, Sahil Srivastava, et al.

Phalguni Mathur, Parveen Kumar Jha, Deepak Kumar, Ajay Acharya, Sahil Srivastava, Pulkit Saxena, Amit Garg, Vishal Dhingra, "Development of low cost optical fiber kit to promote fiber optics at the school level," Proc. SPIE 8481, Optics Education and Outreach II, 84810X (15 October 2012); doi: $10.1117 / 12.946042$

EDIE Event: SPIE Optical Engineering + Applications, 2012, San Diego, California, United States 


\title{
Development of low cost optical fiber kit to promote Fiber-Optics at the school level
}

\author{
Phalguni Mathur, Parveen Kumar Jha, Deepak Kumar, Ajay Acharya, Sahil Srivastava, \\ Pulkit Saxena, Amit Garg, Vishal Dhingra \\ Department of Electronics, Acharya Narendra Dev College, University of Delhi, Govindpuri, \\ Kalkaji, New Delhi-110019, India \\ Email:amit_andc@yahoo.co.in
}

\begin{abstract}
India has a population of more than 770 million mobile subscribers with number increasing at a swift rate. Most of the service providers have shifted to optical technologies based on fiber optics. Though, we have subscribers to various telecom services starting from the school level but they have little idea about the fiber optic technology used. The present development is an attempt to design a "Low cost optical fiber kit" which will explain the basics in a simpler and uncomplicated way. Some of the experiments which are planned to be developed and demonstrated include that on determination of numerical aperture, bending loss, fiber to fiber alignment losses, wavelength division multiplexing, process of optical communication etc. These kits have been developed under University of Delhi at ANDC SPIE student chapter's optics outreach program. The kits will be demonstrated by the chapter student members at various schools in Delhi and distributed free.
\end{abstract}

Keywords: Fiber Optic Kit, Optical Fibers, Numerical Aperture, Fiber losses, Wavelength Division Multiplexing

\section{INTRODUCTION}

With continuous advancements in technology, the present society has learnt the importance of light in the communication domain. The technology underlying the rapid development of internet and mobile services is based on "fiber optics", modern electronic gadgets of ultra-importance today like television, laptops, fax and photocopy machines, scanners, computers etc. also are based on photonics. At the grass root level, the "fiber optics" technology is based on the principle of "Total Internal Reflection". Though total internal reflection is a concept introduced to the students in the schools itself [1], the students still lack both knowledge and the interest in this technology. One of the main reasons is the teaching is more bookish than hand-on through experiments. Hence the students hardly understand the concept and its importance. If this conceptual teaching is supported by experiments and some applications then it will not only help in the understanding but it will also generate the interest among the students towards this domain.

As far as fiber optics is concerned, its trainer's kits are available in the laboratories but more for undergraduate level or above. Those kits cannot be used by the school students as they are complicated and their appearance is enough to demoralize the students at large. They are also very expensive which means that the schools in the rural India can't afford such equipments.

If low-cost and uncomplicated, user friendly fiber optics kits are developed then every science student can not only understand this wonderful technology but also pursue the subject with interest. Our objective was to develop such trainers' kits which will help the students to understand the importance of fiber optics and popularize this area of technology starting from the school students. Our team consisting of the undergraduate students who are members of the University of Delhi at ANDC SPIE student chapter has attempted to develop such low-cost fiber optics kit for school level. Also, the kit developed is very cheap costing around USD 200 and can be easily procured by the schools in cities as well as in the rural parts of the country.

Optics Education and Outreach II, edited by G. Groot Gregory, Proc. of SPIE Vol. 8481

84810X · ( ) 2012 SPIE · CCC code: 0277-786/12/\$18 · doi: 10.1117/12.946042 


\section{METHODOLOGY}

We adopted hands on approach to make students understand how signals are transmitted over different regions using optical fibers. In our project, initially we targeted the students of $12^{\text {th }}$ grade from one of the schools for the demonstration of the kit. We started first by the general introduction of fiber optics and the importance it holds today. A pre test was taken before the beginning of the demonstration. We divided the students into two batches and cyclically changed the batches for demonstration purposes. One of the team members handled the pre and post tests whereas the other three instructors did demonstration of the experiments involving the determination of numerical aperture, the losses associated with the fibers, optical communication and WDM (wavelength division multiplexing) etc. After the demonstration, a post test was taken to measure the success of the workshop. Experiments were done in a very basic manner so that the students can relate them very efficiently and with interest.

\section{FIBER OPTICS KIT FEATURES}

The low cost fiber optics kit developed by our team and used for the demonstration of the experiments has the following components:

$>$ The fiber optic unit

$>$ Optical fibers: $30 \mathrm{~cm}, 1 \mathrm{~m}, 3 \mathrm{~m}$ and assorted fibers

$>$ WDM: acrylic rod and set of coloured filters

$>$ Set of four fiber optics LEDs(Red, Blue, Green and Infrared)

$>$ Fiber optic Detector

$>$ Losses Measurement: Microbend deformer for bending losses, Fiber holder mounts for alignment losses

$>$ Speaker, Microphone, Headphone

The contents of the kit are as shown from Fig. 1(a) to 1(c)

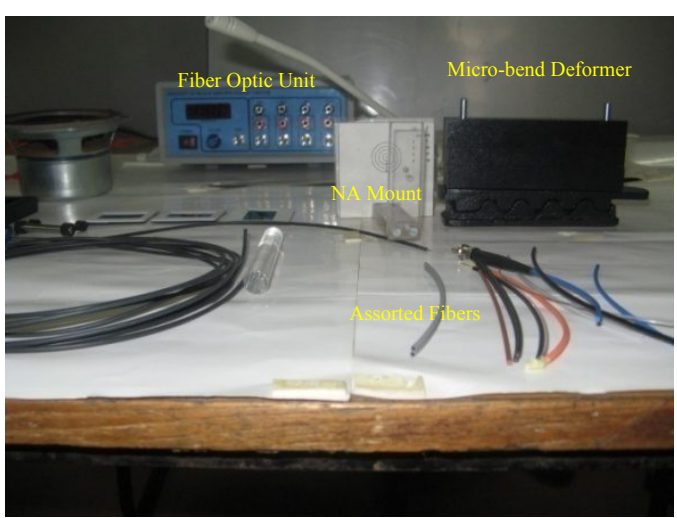

Fig. 1(a)

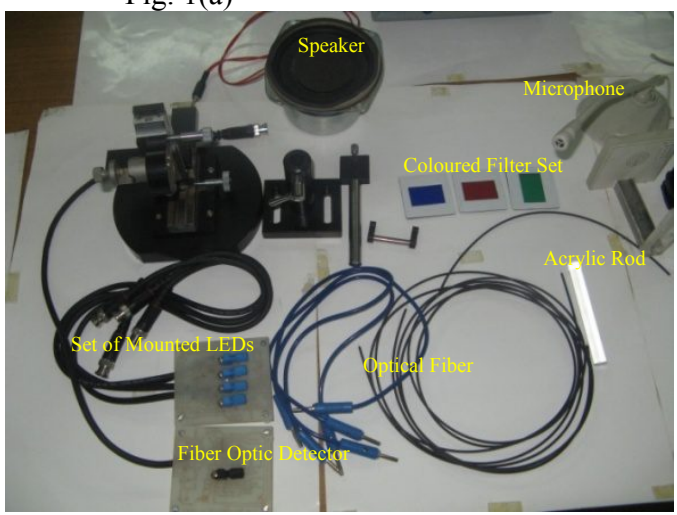

Fig. 1(c)

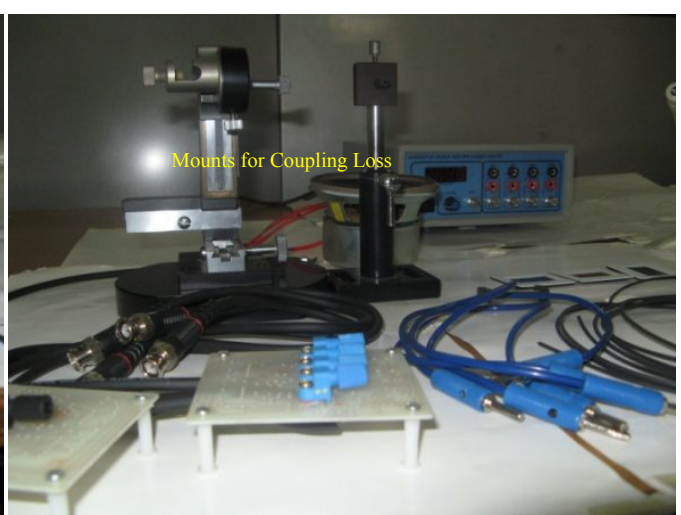

Fig. 1(a)

Fig. 1(a)-(c): Components of Fiber Optics Kit 
Fiber Optic Unit: The kit developed by our chapter members is very basic in terms of electronic circuits involved. The basic purpose of this unit is to derive the four fiber optic (FO) LEDs and amplify the signal received from the fiber optic detector. The voltage output of the detector is also displayed on the panel meter in $\mathrm{mV}$. The developed unit is as shown in Fig.2 (a) with its schematic in Fig. 2(b). There are four outputs Sig1, Sig2, Mic and Pulse available from the kit. Sig1 and Sig2 are the two analog outputs generated using Wien Bridge Oscillator whereas the pulse has been generated using astable multivibrator. The signal from the microphone is amplified using a power amplifier based on LM386 and is available at Mic output (Fig. 2(c)). The set of four FO LEDs can be connected to the unit through the BNC and can be derived using any of the four output signals. Generated sinusoidal, square and/or voice signals modulate three visible LEDs and one IR LED. Fiber optic detector can be connected to the unit through BNC. This signal after amplification using a LM386 power amplifier (Fig. 2(d)) derives the speaker and can also be measured through digital voltmeter. The gain can be adjusted based on the requirement using the potentiometer. The circuits can be designed using reference [2]. The FO LEDs and detector are mounted in such a way that the detector can be placed in front of any of the LEDs (Fig. $2(\mathrm{e})$ and $2(\mathrm{f}))$.

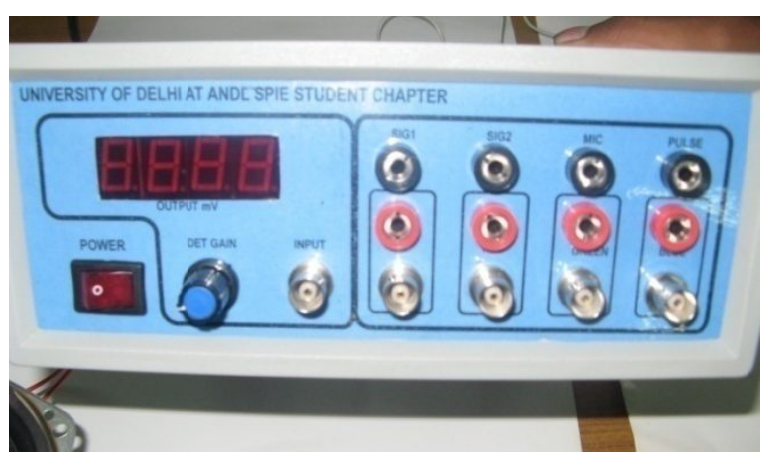

Fig. 2(a): Developed Fiber Optic Unit

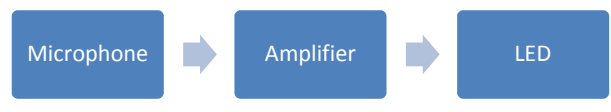

Fig. 2(c): Developed Fiber Optic Unit

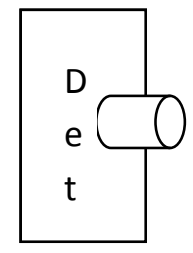

Fig. 2(e): Mounted FO Detector

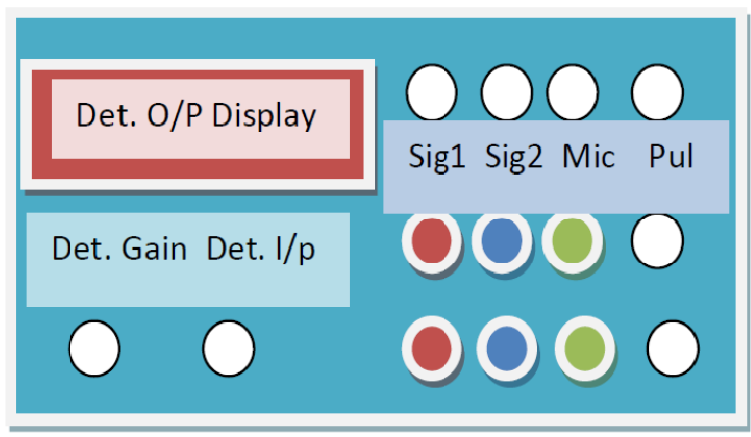

Fig. 2(b) Schematic of the Unit

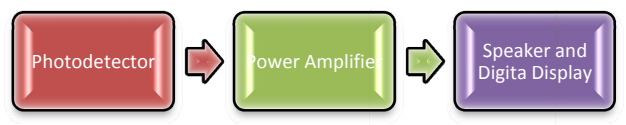

Fig. 2(d) Schematic of the Unit

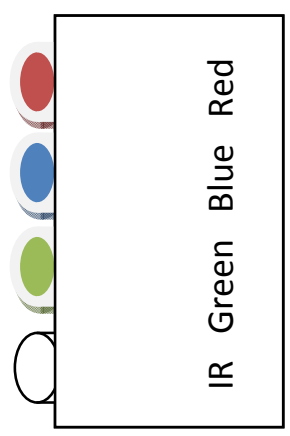

Fig. 2(f) Mounted FO LEDs

The experiments that can be performed using this kit (Fig. 3(a)-3(f)) are as follows:
i. $\quad$ Determine NA of a fiber
ii. Determine bending losses in a fiber
iii. Determine alignment losses in a fiber 
a. Lateral( vertical and horizontal)

b. Longitudinal

iv. Demonstrating various types of fibers and connectors

v. Need for Optical communication

vi. Need of Optical fiber in communication

vii. Demonstrating analog communication using optical fiber

viii. Demonstrating digital communication using optical fiber

ix. Demonstrating voice communication

x. Demonstrating Wavelength Division Multiplexing and its importance

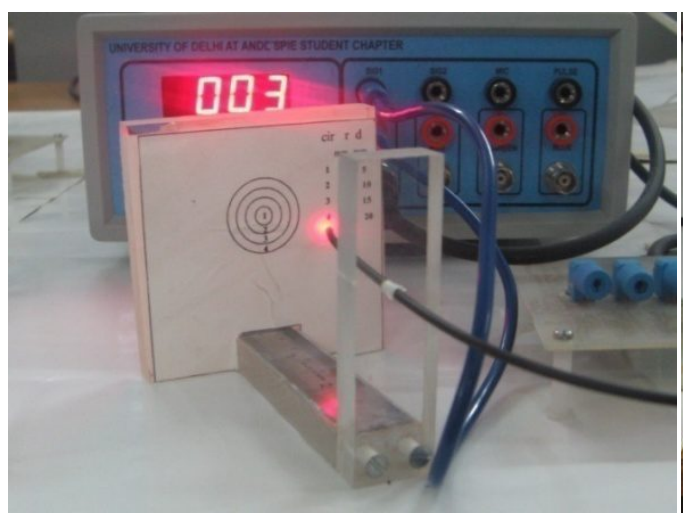

Fig. 3(a): Determine NA of a fiber

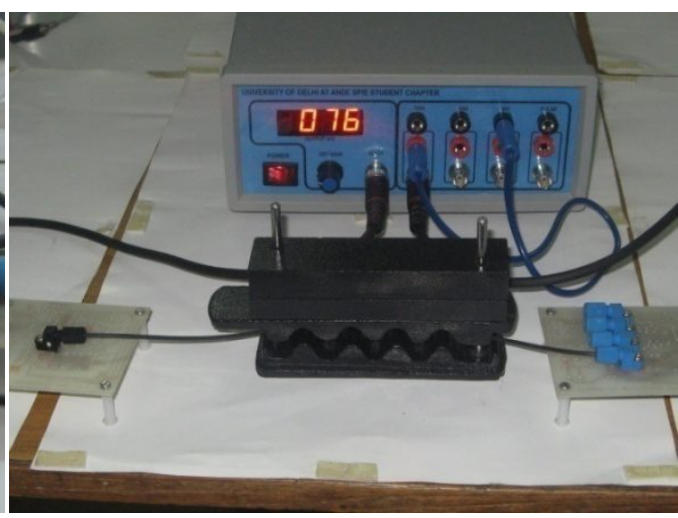

Fig. 3(b): Determine bending losses in a fiber

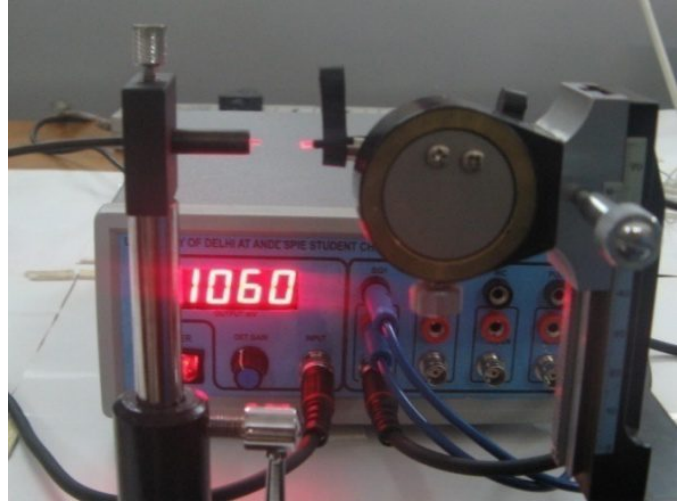

Fig. 3(c): Determine alignment losses in a fiber (Lateral (Vertical and Horizontal) and longitudinal

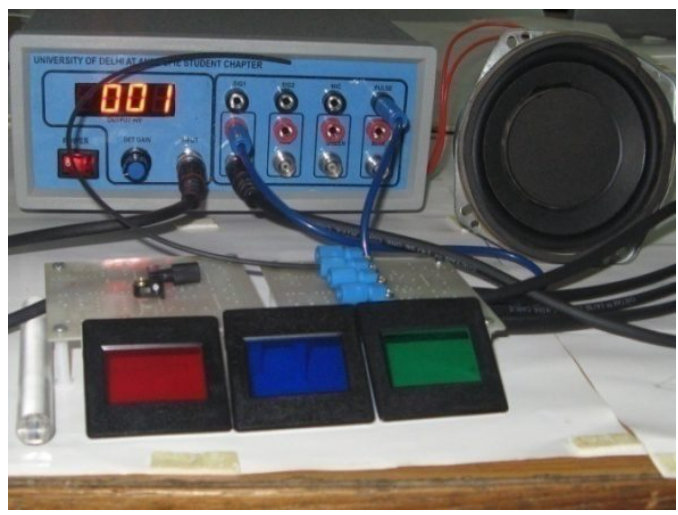

Fig. 3(e): Set up for demonstrating WDM

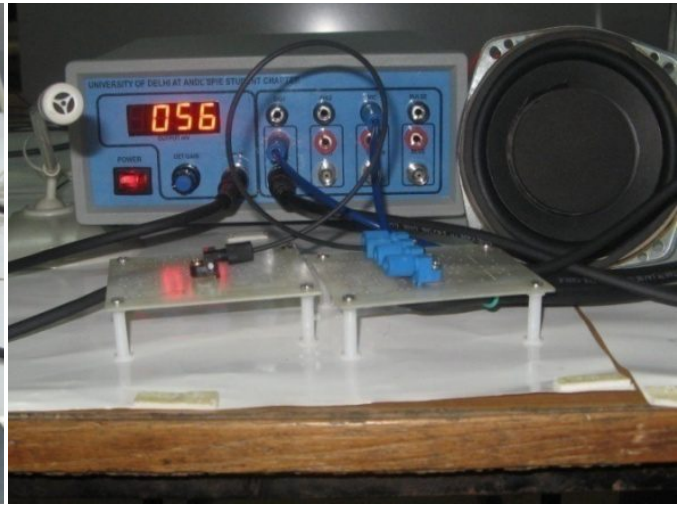

Fig. 3(d): Demonstrating Optical Communication (Analog, Digital and Voice)

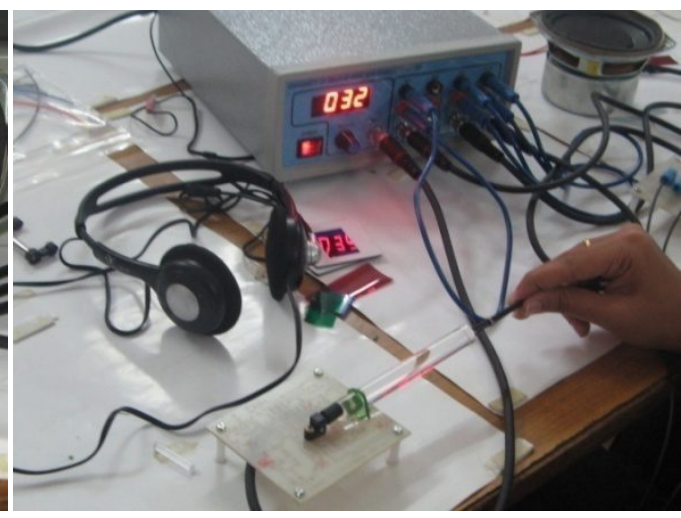

Fig. 3(f): Coupling of the three signals through acrylic rod to demonstrate WDM 
For bending losses measurements, a microbend deformer has been used. The fiber is pressed between the two wavy faces of it and microbends are produced in the fiber by increasing the weight on its upper face. The changes in the light intensity through the output end of the fiber are shown on the FO unit display. For measuring the coupling losses, two low cost coupling stages are used. The input stage holds the fiber straight whereas the second stage has the provisions for lateral as well as longitudinal translation. Further, the lateral movement is possible both along horizontal and vertical directions. Thus, it is possible to show both lateral and longitudinal losses.

For demonstrating wavelength division multiplexing (WDM), the RGB FO LEDS are derived from the three signals and are coupled to the acrylic rod which acts as an optical multiplexer. The acrylic rod is aligned to the FO detector making all the three wavelengths falling on to it. Filter of respective colour is inserted between the rod and the detector to demultiplex any of these signals.

\section{RESULTS \& DISCUSSIONS}

The workshop started with a small introductory talk about the fiber optics and their significance today.The initial understanding of fiber optics among the students was measured by short pre-test containing simple multiple choice questions about fiber optics.

The questions raised were as follows:

1. What does IR LED stand for?

2. What is the most common material of which the optical fiber is made of?

3. On which phenomenon fiber optics is based?

4. Which are the two most common sources used in fiber optics?

5. What is the refractive index of fiber material with respect to air?

6. How are wavelength and frequency of a light wave related?

7. What among the following are the important components of optical fiber communication?

8. Optical fibers transmit energy in what form?

9. Which among the following is the application of optical fiber communication?

10. Is it true that optical fibers can carry signals with very less power loss as compared to the copper cables?

11. What does the numerical aperture of the optical fiber define?

12. Will optical fiber allow light to escape from sides?

13. What do you understand by wavelength division multiplexing?

14. What do you understand by bending losses in optical fibers?

15. What do you understand by fiber to fiber alignment losses?

After the pre test, we started the demonstrations showing the various types of fibers, connectors available in the market followed by the listed experiments. A post test was then conducted to measure the effectiveness of our developed kit in explaining various concepts related to fiber optics. The post test contained the same questions as in the pre test. Fig. 5 shows the results we obtained after going through the two tests:

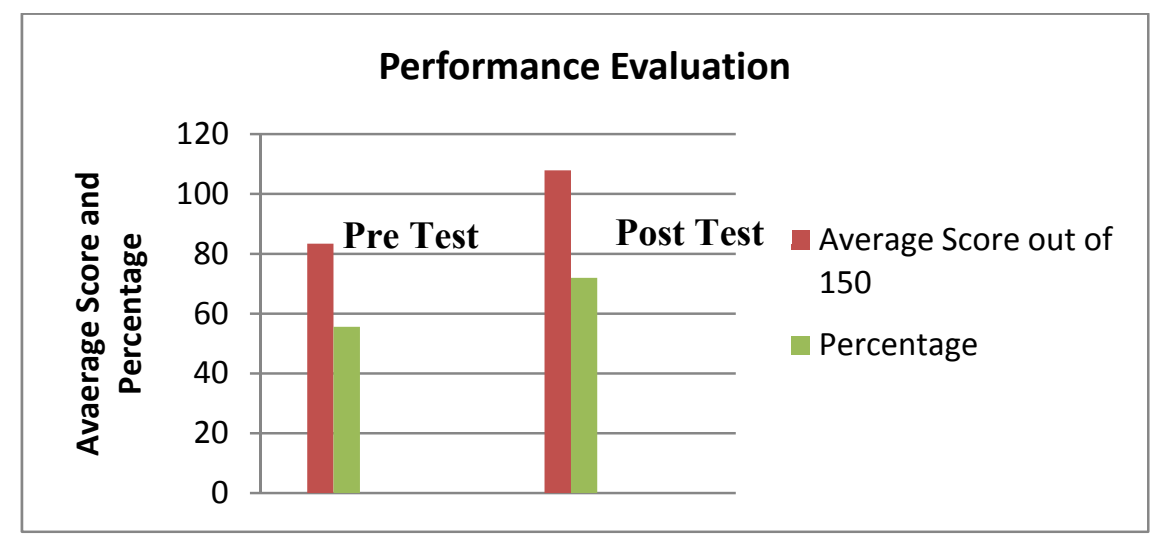

Fig. 5: Performance of students in Pre and Post test 
As shown in Fig. 5, there is a pronounced improvement in the understanding of the students after demonstrations. Further, this is what the Head, Department of Science of the school has to say about the demonstration and its impact on the students

The students of Acharya Narendra Dev (AND) college were welcomed by the blank expressions and seamless stares from the students on the announcement of the topic but as the workshop progressed so did the interest levels among the students. A pre test regarding optics was passed on in the class and following the workshop, a post test was conducted.

The workshop was a table turner for students, who at first had thought of optical fibers to be something related to complex calculations and theorems now understood it to be based on a far simpler phenomenon and also its vast usage in the days to come. Explaining everything to the students starting from square one was tedious and required immense patience which was portrayed by the students of AND and they succeeded in capturing our attention towards SPIE and optical fibers.

Images of the team members conducting the demonstrations is as shown in Fig. 6(a)-6(f).

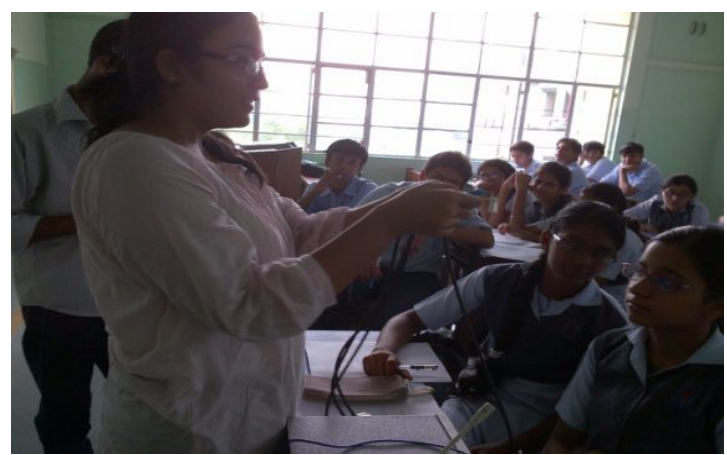

Fig. 6(a)

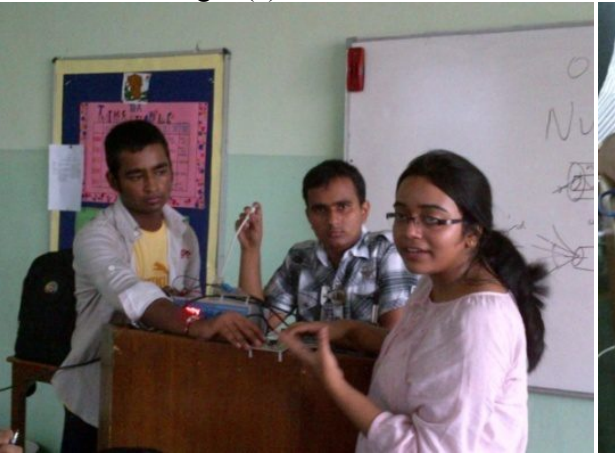

Fig. 6(c)

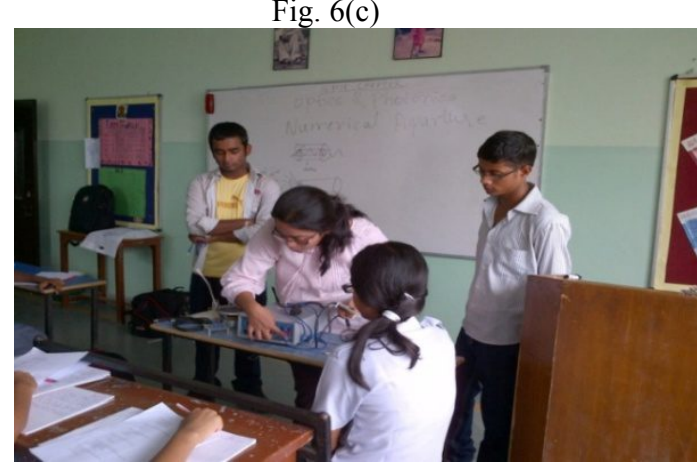

Fig. 6(e)

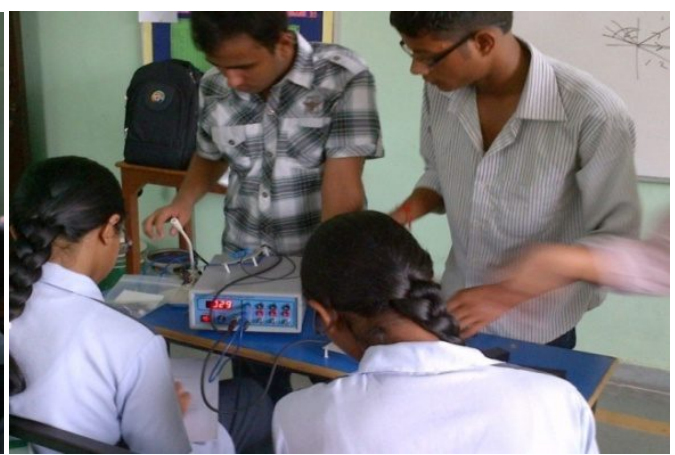

Fig. 6(b)

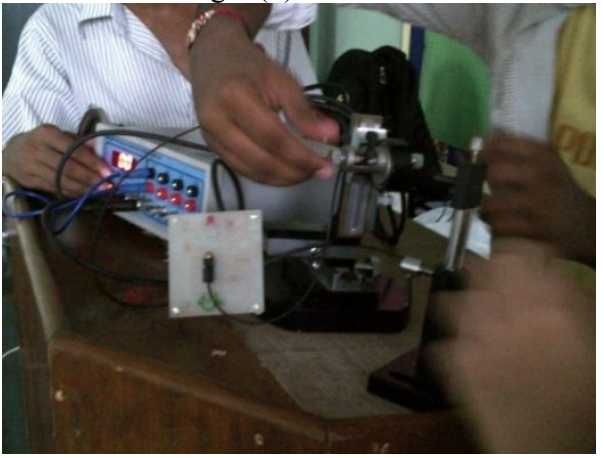

Fig. 6(d)

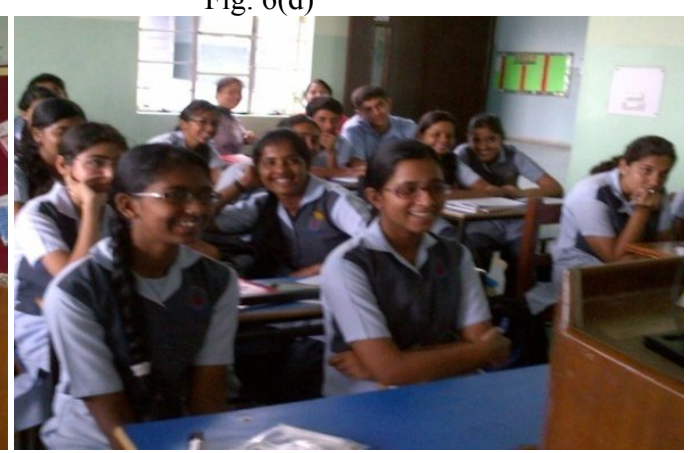

Fig. 6(f)

Fig. 6(a) - 6(f): Photographs of the team members conducting the demonstration of the FO Kit and towards the end the happy faces of the students 


\section{CONCLUSION}

The kit developed gave the students hands- on experience about various characteristics/features of optical fiber and how exactly signals are transmitted. They also came to know exactly why fiber optics is so very important in our day to day life and catering our current bandwidth demands. It also made it easier for the students to learn the concepts involved in fiber optics and help in enhancing their skills. The experiments performed have definitely sparked the interest in them towards this wonderful technology.

\section{ACKNOWLEDGEMENT}

One of the authors Phalguni Mathur would like to thank SPIE, USA for providing Officer's Travel grant to attend and present this paper in SPIE Optics + Photonics 2012 conference from $12^{\text {th }}-16^{\text {th }}$ August 2012 in San Diego, California, USA.

\section{REFERENCES}

1. http://www.ncert.nic.in/NCERTS/textbook/textbook.htm?leph2=1-7

2. R. A. Gayakwad, Op-Amps and Linear Integrated Circuits, Pearson Education (2003) 